\title{
E-C Translation of Medicine-Health Texts: Optimal Relevance
}

\author{
Wenmin $\mathrm{Li}^{*}$, Jie Qian, Kangting Zhang \\ School of Arts and Sciences, Shaanxi University of Science and Technology, Xianyang, China \\ Email: *yezi_wm66@163.com
}

How to cite this paper: Li, W. M., Qian, J., \& Zhang, K. T. (2018). E-C Translation of Medicine-Health Texts: Optimal Relevance. Open Journal of Modern Linguistics, 8, 294-303.

https://doi.org/10.4236/ojml.2018.86023

Received: December 3, 2018

Accepted: December 26, 2018

Published: December 29, 2018

Copyright ( $) 2018$ by authors and Scientific Research Publishing Inc. This work is licensed under the Creative Commons Attribution International License (CC BY 4.0).

http://creativecommons.org/licenses/by/4.0/ c) (i) Open Access

\begin{abstract}
Translation activities, in the context of globalization, promote communication between people from different countries with different languages, and enhance the fusion of the two cultures behind these two languages concerned. This paper, focusing on the ostensive-inferential communication in translation process of the medicine-health text named Your Medical Mind: How to Decide What Is Right for You, aims at achieving successful communication between the authors of this paper and their target readers. By concrete analysis of the target text from the lexical level, syntactic level and cultural level, this study finds that it is the translators who take the most active and key role to bridge the two languages and cultures for communication, and only under the target readers' good comprehension of the meaning conveyed by the source text can the translators succeed in transmitting the informative intention and communicative intention through ostensive stimulus to their target readers.
\end{abstract}

\section{Keywords}

Medicine-Health Texts, Relevance Theory, Optimal Relevance,

Ostensive-Inferential Communication, E-C Translation

\section{Introduction}

With the boom of the world economical and cultural communication, the health awareness of Chinese people is rapidly strengthened with the rapid development of science and technology which has opened up a new era of the world's attention to the medicine and health care as well as the worldwide sharing of medicine and health care. Chinese people are curious about what the foreigners, especially people from English-speaking counties, do about their health. Thus it is vital for the translators to boost the research on foreign advanced medi- 
cine-health related knowledge and the relevant studies on E-C translation of medicine-health texts.

Relevance theory, though belonging to the cognitive pragmatics, is powerful and systematic enough to guide the translation industry. According to relevance theory, translation is an ostensive-inferential communication process in which optimal relevance to the source text as well as the target cultural context is sought (Gutt, 1991: p. 20).

Currently, most studies on Relevance Theory focus on the translation of literary works, advertisements and subtitles. There are few papers talking about the application of Relevance Theory in medicine-health texts. Hui Jiabei (2011) sought optimal relevance anticipation in culture-specific lexicons in Shang Han Lun based on English versions. Zhao Tingting (2013) analyzed the translation of disease treatment in The Story of the Stone from the perspective of relevance translated theory. Li Di (2014) made the comparison between the original meaning of the source text, the context of the target reader and the expression of the target text in E-C Translation of Medical English from the Perspective of Relevance Theory. Zhang Miao (2017) studied the culture-loaded words in Huangdi Neijing with optimal relevance. This paper aims to discuss the guiding role of Relevance Theory in medicine-health texts from the perspective of seeking the optimal relevance.

\section{Medicine-Health Texts}

\subsection{The Chosen Source Text}

Medicine-health texts of whatever language belong to scientific and technical texts. Though, language in this kind of text has its own distinctive characteristics due to distinctive cultures. Understanding the characteristics of medicine-health texts of a certain language, which concerns a good master of both the language and its culture, is of great importance to well comprehend and precisely translate them into another language. The authors make some case studies on some chosen chapters from the book named Your Medical Mind: How to Decide What Is Right for You which perfectly shows the characteristics of medicine-health texts in English. And the authors have chosen this book as E-C translation practice.

Your Medical Mind: How to Decide What Is Right for You is written by Jerome Groopman and Pamela Hartzband. Jerome Groopman is not only a New Yorker writer and bestselling author, but also an oncologist who gives his patients suggestions on lifesaving decisions. Pamela Hartzband is a noted endocrinologist and educator at Harvard Medical School who helps patients make critical decisions about their long-term health. The authors in this book convey groundbreaking message: everyone, being a patient instead of a medical expert, can solve the confusion out and find the most suitable decision about treatment. The idea of the medical mind is new and encouraging to people, especially to us Chinese who have no idea but count completely on their doctor while getting sick. 


\subsection{The Characteristics of the Source Medicine-Health Texts}

Since medicine-health texts combine medicine and health, it is both informative and communicative. On the lexical level, there are many terminologies. And in this kind of text, the authors of the book adapted narratives to convince the patients of their ideas. It is also key to pay attention to the coherence between each two sections of the source medicine-health texts, especially the coherence by conjunctions. On the syntactical level, as the common features in medicine-health text, there are many long, complex sentences and passive-voice sentences.

In recent years, with the improvement of people's living condition and popularity of science and technology, people pay more attention to their health. According to the present status, there are ever-increasing E-C translation of medicine-health concerned books in China, especially Chinese medicine oriented health care books. Moreover, it's more functional for Chinese people to introduce books concerning scientific and medical expert-guided ideas and information from abroad.

\subsection{The E-C Translation of the Medicine-Health Texts}

It is a must to translate the medicine health book from English into Chinese, for Chinese people badly need it with ever increasing awareness of their body health. As for the requirements, firstly, what the source text tells should meet the need of the target readers. Secondly, the translator should follow the principle of objectivity from the informative perspective of medicine health text, and the target text should be precise and authentic in information. Thirdly, the target text should also be readable, infectious and convincing in communication. Only with the three points mentioned above is the translation a good target text.

\section{Literature Review}

\subsection{Relevance Theory}

Starting from Grice's ambiguous definition "Be relevant" for the maximum of Relation in Cooperative Principle, Relevance Theory treats Relevance as a property of inputs to cognitive processes (Hou, 2006). Relevance theory was proposed by the French linguist, Dan Sperber, and the English linguist, Deirdre Wilson, in the book Relevance: Communication and Cognition in 1986. In 1989, Ernst Gutt, their student, firstly applied Relevance Theory in translation in Translation and Relevance: Cognition and Context. Relevance Theory holds the opinion that the communication process involves not only encoding, transfer, and decoding of messages, but also numerous other elements, including inference and context. It focuses on ostensive-inferential communication mainly involving informative intention, communicative intention and ostensive stimulus. In this communication process, the author conveys as much information by way of ostensive stimulus in a given context in which the audience is so much concerned so that the author intends to create the mutual cognitive environment 
to help his or her reader recover his or her intended meaning. That is to say, the author purposefully gives a clue to the audience ("ostensifies"), as to what he or she wishes to communicate-that is a clue to his or her intention. The reader infers the intention from the clue and the context-mediated information. The reader must interpret the clue, taking into account the context, and surmise what the author intended to communicate.

\subsection{Cognitive and Communicative Principles}

Sperber and Wilson believe that cognitive principle and communicative principle are two basic ones that people should abide by in effective communicative activities. In other words, all the cognitive and communicative activities focus on relevance of the two parties involved in communication. There are two general principles of relevance, for the relevance means separately different to cognition and communication (Sperber \& Wilson, 1995). Cognitive principle means that Human cognition tends to be geared to the maximization of relevance while Communicative principle meets optimal relevance.

\subsection{Optimal Relevance}

Sperber and Wilson hold that the relevance degree relies on two factors: contextual effects and processing effort, and it is a matter of balancing contextual effects against processing effort. Contextual effects are important factors in assessing the degrees of relevance: "We want to argue that having contextual effects is an essential condition for relevance, and that other things being equal, the greater the contextual effects, the greater the relevance" (Wang, 2000: p. 13). In addition to contextual effects, another factor, the processing effort is the second important factor to be considered in assessing degrees of relevance: "Processing effort is a negative factor: other things being equal, the greater the processing effort, the lower the relevance" (Wang, 2000: p. 13).

According to the core of Relevance Theory, optimal relevance should be achieved in translation process (Meng, 2002), and it requires that the translation provide sufficient contextual effects so that the target readers, with less processing efforts, better comprehend the intended meaning the translator conceives in the target text. A formula could illustrate the relation among the optimal relevance, contextual effect and processing effort: the optimal relevance $=$ contextual effect/processing effort (Zhao, 2003).

In translation process, the translator is seeking for the target of the optimal relevance so as to satisfy their target readers to the most with his translation. The task of the translator should try to make the intention of the author and the expectation of the target readers consistent (Lin, 1994: p. 7).

\section{Application of Optimal Relevance in E-C Translation of the Book}

Optimal Relevance can be employed to the chosen text-Your Medical Mind: 
How to Decide What Is Right for You due to its property of medicine-health. The authors create an ideal context in which the potential target readers are expected to achieve optimal relevance with the least processing efforts in their communication with both the source authors and the target readers.

The authors in this paper take dual identity. On one hand, the authors, as the readers of the source text, should correctly understand the original intentions of the authors and speculate their ideas intended in the source text. On the other hand, the authors, as the translators of the source text, should create a mutual cognitive context in which the ideas they conveyed from the source text can be better understood by the target readers.

First of all, the authors need to judge whether the context the original authors create in the source text to the source readers exists in the target one, and it requires that the authors configure a cognitive context for the target readers in translation process, by finding out in which way the target readers expect to accept the information conveyed and making sure the target text ideal to the target readers. Secondly, the authors should judge whether it is difficult for the target readers to understand the target cognitive context and whether the processing effort corresponds with the target cognitive context. It requires that the authors achieve the optimal relevance by increasing the contextual effect and decreasing the processing effort.

How, then, could the authors, if they don't share the same background of culture, achieve the same equivalence in their target text? Since the authors of the source text have their own background and must have their own intention expressed in the source with their potential readers. Dr. Jerome Groopman and Pamela Hartzband, authors of Your Medical Mind: How to Decide What Is Right for You are American medical experts and both have the experience to give their patients suggestion to make self decisions in life saving. Their potential readers are both the American patients who have no ideas but turning to their doctors when getting sick and those who care much about their health. That's why this book was one of the bestselling books in New York. The authors of this paper, the translators of this book are Chinese, and they are the student of Master of Translation and Interpreting and her supervisor. They are translating it for the intention to improve the awareness with self help of the Chinese people who are getting sick or who care much about their health.

How could the authors or the translators, as non medical experts, achieve the same functionally dynamic equivalence in the translation version of medicine care target text so as to satisfy target readers? In translation process of this book, the authors or the translators read three other medicine care related books named Marketplace of the Marvelous: the strange origins of modern medicine, Disease Proof: The Remarkable Truth about What Makes us Well, Goddesses Never Age: The Secret Prescription for Radiance, vitality and wellbeing, which are all American medical books. And they also read some Chinese medicine care related books such as Only Doctors Know it and What you eats Influences How 
you live., the authors of which respectively are Chinese doctor and nutritionist. Secondly, they made comparison of language in the above books between English and Chinese. Reading these books enables them to be familiar with the style of medicine care related texts and be aware of the use of language both in English and Chinese. And they made comparison between the potential readers from both the source text and the target text for a better understanding of the similarity and difference between them. Finally, they tried their best to create the ideal context and choose the proper words and expressions to achieve the optimal relevance on lexical, syntactic and cultural level.

\subsection{The Optimal Relevance on Lexical Level}

It is undoubtedly that as texts concerning about medicine-health, terminology matters a lot to the authors. Besides, conjunctions are widely used in English expressions. To tell the difference between various conjunctions is also a major problem, for it usually represents different meanings in different context. Thus referring to the co-context contributes to the understanding the intention of the original authors.

\subsubsection{Terminology}

Medical terminology is one of the features in medicine-health texts. Generally speaking, it is hard for the target readers to understand the meanings of terminologies without annotations to them. Therefore, according to different context, the authors need to adopt different methods translation process.

E.g. 1 Aspirin, Lipitor, Crestor, Zocor

Translation: 阿司匹林, 立普妥, 瑞舒伐他汀, 辛戈他丁(治疗高胆固醇的常 用药)

E.g. 2 I remembered what happened a few years ago with Vioxx, how excited everyone was about it and how doctors said it was so much better than aspirin and other drugs.

Translation: 我记得抗炎症药万络, 起初所有人都很振奋, 医生也认为这比 阿司匹林之类的药更加有效, 后来发现它同样会引发心脏病。

In example 1, all the terminologies have already had their Chinese equivalence before the translation of this book, which made the translation of the terminologies easier by dictionaries. All the terminologies mentioned in above are transliterated, but, unlike Aspirin which is very familiar for its wide use to almost all the potential readers, Lipitor, Crestor, Zocor are not so popular unless the readers are patients of cholesterol. That's why the authors chose to add annotation in transliteration of the above words, so that they achieve better contextual effect than they should without annotation, and it is easy and comfortable for the target readers to understand them. In example 2, "Vioxx" is an anti-inflammatory agent. In this sentence, the authors specified the function of the medicine for the target readers so that they can understand it with minimum efforts. Thus they achieved the optimal relevance in translation. 


\subsubsection{Conjunction}

Conjunction words are frequently used in the book. It is hard to figure out the intention of the authors with the conjunction words. For instance, "and", sometimes is translated into “和”, while sometimes into “却” in Chinese. Take “My father lived with a cholesterol level just like mine, and never took a pill" as an example: the speaker in the source text expresses her unwillingness of taking pills. According to the common sense, people who live with a cholesterol level should take pills. But actually, the speaker's father who lived with a cholesterol level never took a pill. So the authors get the exact meaning implied in the word "and". "And" here, as a conjunction, is similar with "but" in meaning. The authors then, as the translators of the source text, convey the exact meaning of the word "and" they comprehend from the source context to the target readers in the target text as “却”. Therefore it is wise to translate "My father lived with a cholesterol level just like mine, and never took a pill” into “我的父亲和我一样 患有高胆固醇，却从未服药”. The word “却” was chosen to achieve contextual effect so that the target readers could get the optimal relevance with the least effort. The successful communication can't be achieved otherwise.

\subsection{The Optimal Relevance on Syntactic Level}

Source medicine-health text widely adopts long, complex sentences. The reason why using these kinds of sentences is that medical English focuses on precise expression of the logic of everything. Completely conveying and stressing the complex and exact messages matters the most in communication. No parts that may confuse and mislead the readers should exist, or it may lead to medical faults on patients. On the other hand, passive voice is often preferable in source medicine-health text because of the long and complex sentences.

\subsubsection{From Long and Complex Sentences into Short and Simple Ones}

In medicine-health text, the most difficult problem is the long, complex sentences. To make readers understand the meaning of the source text, the authors chose to divide the long sentences into one main simple sentence accompanied with several short phrases. For example, "The cardiologist recommended that he wear a heart monitor throughout the day, which showed that he was still having episodes of the art normal rhythm, even though he didn't realize they were happening”. It is a compound sentence, and the authors translated it first into “心脏 病科医生建议他 24 小时背带心脏监护器, 即使他感觉不到任何异常, 但是监 护器可以监测到他的心律异常时段”. If this sentence were translated back into English, it would be like this: "Thecardiologistrecommendedthatthroughoutthedayhewearaheartmonitor which showed that he was still having episodes of the art normal rhythm, even though he didn't realize they were happening". There is the word which in both English sentences, but which means sharply different. Therefore, the translation misled the target readers and the optimal relevance failed in this way. The exact translation should be “心脏病科医生建议他全天佩 带心脏监护仪，这表明他仍然有心率异常现象，即使他没有意识到这种情况 
在发生”. It's no easy job for the authors to create an ideal context if he fails to command full understanding of the source text.

\subsubsection{From Passive-Voice Sentences into Active Voice Ones}

Passive voice is commonly used in English expressions, for it represents objective orientation. However, it is hard for readers to judge what the subject of the sentence is if passive voice is used. As a result, the authors should shoulder the task of distinguishing the subject in translation process.

E.g. 1 When the Royal Canadian Air Force exercise program was published in 1961, he became a fan and encouraged my sisters and me to follow the program with him, carefully measuring our progress.

Translation: 1961 年, 政府颁布加拿大皇家空军锻炼计划时, 父亲十分热衷, 并且鼓励我和妹妹们跟着他一起锻炼, 时刻关注我们的进步。

In the above example, finding out the subject is the primary task, since it is a sentence of passive voice. It's true that only the government has the power to publish a program like that. Thus, here the authors adopted amplification to make up for “政府” as the subject. Thanks to the subject supplemented, the target readers do not need to pay any more processing effort to think about the subject, as well as achieve the maximum contextual effect. That is the way of translation achieving the optimal relevance.

\subsection{The Optimal Relevance on Cultural Level}

\subsubsection{Influence of Cultural Default}

E.g. $1 \mathrm{My}$ father also had high cholesterol, and he died after a long and healthy life, without taking any medication

Translation: 我父亲同样胆固醇过高, 虽然没有使用药物治疗, 但是他生前 依旧高寿而健康。

Cultural discrepancy commonly appears in translation process due to different ways of thinking and cultural background, hence, sometimes literal translation is not suitable for every case. In example 1, "My father also had high cholesterol, and he died after a long and healthy life, without taking any medication”, the authors translated it into “我父亲同样胆固醇过高, 虽然没有使用药 物治疗, 但是他生前依旧高寿而健康”. “高寿” is a commonly used word concerning health in Chinese culture, so the authors replaced general meaning “长” or “漫长” with “高寿” which suits Chinese context and appeals to target readers. What's more, the authors are non medical experts, short of medical experience and medical knowledge. Well comprehending the complicated culture behind the language matters most to the authors in translation so as to make the target text achieve the optimal relevance by achieving the same contextual effect and making the target readers pay the minimum effort.

\subsubsection{Influence of Cross-Cultural Factor}

E.g. 2 Outside the United States, endocrinologists favored antithyroid drugs.

Translation: 美国以外国家和地区的专家赞成使用抗甲腺药剂。 
Attention should also be paid to political reasons both in cultural communication and in translation. It can be seen in the example 2 that to achieve the optimal relevance, the authors should add “国家和地区” to the translation. Because in the world, there are not only countries, but also regions such as Taiwan and Hong Kong. The target readers will not spend more time considering whether there are any regions besides countries outside the United States. The authors flatly pointed out the detailed messages for them. Thus the target readers could receive the same expectations as what the source readers expect.

\section{Conclusion}

No matter what technique, semantic translation or communicative translation, is employed in translation, the only purpose for the authors is to pass the information from the source text to the target readers. It's essential to achieve optimal relevance by increasing the contextual effect and decreasing the processing efforts. The contextual effect counts on the authors' cognitive capacity in understanding the source text and its authors, and the communicative capacity in building the context. The processing efforts depend on how effective the context created for the potential readers. The only thing insufficient is that optimal relevance cannot be fully tested by its potential readers for the translation version of this book hasn't been published. The contextual effect the authors created has been appreciated by the readers who are students of MTI and teachers of English.

\section{Conflicts of Interest}

The authors declare no conflicts of interest regarding the publication of this paper.

\section{References}

Gutt, E. (1991). Translation and Relevance: Cognition and Context (p. 20). Brookland: St. Jerome Publishing.

Hou, J. Y. (2006). Empirical Research on the Cognitive and Communicative Principle. Master Dissertation, Changsha: National University of Defense Technology.

Hui, J. B. (2011). A Comparative Study on the English Translation of Culture-Loaded Words in Shang Han Lun: Based on Two English Versions. Nanjing: Nanjing University of Chinese Medicine.

Li, D. (2014). English-Chinese Translation of Medical English from the Perspective of Relevance Theory-Exemplified by an English Dentistry Text. Changsha: Hunan Normal University.

Lin, K. N. (1994). Brief Introduction to Relevance Translation Theory. Chinese Translation, No. 4, 6-9.

Meng, J. G. (2002). Optimal Relevance of Translation Principles of Duality. Chinese Translation, No. 5, 27-31.

Sperber, D., \& Wilson, D. (1995). Relevance: Communication and Cognition (p. 260). Oxford: Blackwell Publishers. 
Wang, B. (2000). Limitations of Relevance Theory on Translation. Chinese Sci-Tech Translation, No. 4, 13-16.

Zhang, M. (2017). The Study of the Translation of Culture-Loaded Words in HuangDi NeiJing in the View of the Relevance Theory. Western Journal of Traditional Chinese Medicine, No. 10, 142-145.

Zhao, T. T. (2013). Diseases Treatment in The Story of Stone from the Perspective of Relevance Theory. Shi Jiazhuang: Hebei Normal University.

Zhao, Y. C. (2003). The Essence of Relevance Theory and Translation. Journal of Sichuan International Studies University, No. 3, 117-121.

\section{Appendix}

Christiane, N. (2016). Goddesses Never Age: The Secret Prescription for Radiance, Vitality, and Well-Being. Carlsbad: Hay House.

David, K., \& Stacey, C. (2013). Disease Proof: The Remarkable Truth about What Makes us Well. Boulder: Avery.

Erika, J. (2014). Marketplace of the Marvelous: The Strange Origins of Modern Medicine. Boston: Beacon Press.

Groopman, J., \& Hartzband, P. (2011). Your Medical Mind: How to Decide What Is Right for You. London: Penguin Group. 